\title{
Cue-dependent safety and fear learning in a discriminative auditory fear conditioning paradigm in the mouse
}

\author{
Makoto Takemoto ${ }^{1}$ and Wen-jie Song ${ }^{1,2}$ \\ ${ }^{1}$ Department of Sensory and Cognitive Physiology, Faculty of Life Sciences, Kumamoto University, Kumamoto 860-8556, Japan; \\ ${ }^{2}$ Program for Leading Graduate Schools HIGO Program, Kumamoto University, Kumamoto 860-8556, Japan
}

\begin{abstract}
Discrimination between sensory stimuli associated with safety and threat is crucial for behavioral decisions. Discriminative conditioning paradigms with two acoustic conditioned stimuli (one paired with shock [CS+], the other unpaired with shock [CS-]) have been widely used as an experimental model for fear learning. However, no attention has been paid to the effect of the CS- on safety in the paradigms, because the CS- served as a neutral cue or elevated the freezing level due to fear generalization although less effectively than the CS+. By using a noise and a tone as two acoustic CSs in a discriminative auditory fear conditioning (AFC) paradigm, here we demonstrate that mice learn safety for the CS- while showing fear for the CS+ with opposing emotional behaviors. We found that after learning mice exhibited a significant suppression of context-dependent freezing during the $\mathrm{CS}_{-}$, but not during the $\mathrm{CS}_{+}$, indicating learned safety without fear generalization for the CS-. In contrast, the mice showed an enhanced level of freezing during the CS+ even in a novel spatial context, indicating cued fear for the CS+. Moreover, the CS+ also induced rapid defensive behaviors, whereas the CS- disinhibited normal exploratory behaviors. On the other hand, mice showed no significant suppression of contextual fear during the CS- in a paradigm with a pair of tone CSs at different frequencies, although they clearly discriminated the two tones. These results suggest our AFC paradigm with the noise and tone CSs as a useful experimental model for cue-dependent discriminative learning of safety and threat.
\end{abstract}

Learning to discriminate sensory stimuli associated with safety from those associated with threat is crucial for correct behavioral decisions. Impaired discrimination of the associated emotional valence between these stimuli can lead to fear generalization (Dunsmoor and Paz 2015), a symptom of anxiety disorders observed in a wide variety of neuropsychiatric disorders, such as posttraumatic stress disorder (Etkin and Wager 2007; Jovanovic et al. 2012), schizophrenia (Holt et al. 2012; Braga et al. 2013; Kiran and Chaudhury 2016), and autism spectrum disorders (van Steensel et al. 2011; White et al. 2014). Therefore, experimental models of discriminative safety versus fear learning are beneficial for the research on neural mechanisms of emotional discrimination and generalization, as well as on related disorders (Kheirbek et al. 2012).

As classical experimental models of safety learning, two types of fear conditioning paradigms have been used in rodent studies: contextual fear conditioning with a single acoustic conditioned stimulus (CS) that is associated with a period of time in the absence of aversive unconditioned stimuli (US) (Rogan et al. 2005; Pollak et al. 2008, 2010), and cued fear conditioning with two CSs: one paired with an aversive US (CS+) and the other explicitly unpaired with the US (CS-) (Hammond 1967; Christianson et al. 2012; Sangha et al. 2013; Foilb et al. 2016). A critical point of the difference between these two paradigms is how to evaluate learned safety. In the single-cue paradigm, learned safety is evaluated by a suppressive effect of the CS on contextual fear that is acquired by the aversive US in the conditioning context (Rogan et al. 2005; Pollak et al. 2008, 2010). On the other hand, in the dual-cue paradigm, conditioned safety is evaluated by an attenuating effect of

\section{Corresponding author: takemoto@kumamoto-u.ac.jp} Article is online at http://www.learnmem.org/cgi/doi/10.1101/lm.049577.119. the CS- on CS+-evoked fear when presenting both the CSs simultaneously in the testing session, a test referred to as a summation test (Hammond 1967; Rescorla 1969; Christianson et al. 2012). Importantly, to prevent animal subjects from misunderstanding the superposed CSs as a novel cue, two cues with different sensory modalities, such as sound and light, need to be assigned to the CSs in the dual-cue paradigm (Hammond 1967; Christianson et al. 2012; Sangha et al., 2013; Foilb et al. 2016). Although a retardation test is another way to evaluate learned safety that assesses a low effectiveness of the CS on fear acquisition after the same CS being acquired as a safety cue (Rescorla 1969), safety conditioning and the subsequent reconditioning for fear need to be performed independently (Rescorla 1969; Rogan et al. 2005; Pollak et al. 2008; Christianson et al. 2012; Sangha et al. 2013; Foilb et al. 2016).

A large number of studies have taken advantage of discriminative auditory fear conditioning (AFC) paradigms with two sound CSs for investigating fear learning and memory (Antunes and Moita 2010; Letzkus et al. 2011; Aizenberg and Geffen 2013; Courtin et al. 2014; Ghosh and Chattarji 2014; Botta et al. 2015; Abs et al. 2018). However, although the effect of the CS+ on conditioned fear has been assessed by comparing fear intensities between the CS+ and the CS-, based on an increase in freezing responses above the baseline level, no attention has been paid to the effect of the CS- on safety learning. This is probably because the freezing level was either unaffected by the CS- (Ghosh and

\footnotetext{
(C) 2019 Takemoto and Song This article is distributed exclusively by Cold Spring Harbor Laboratory Press for the first 12 months after the full-issue publication date (see http://learnmem.cshlp.org/site/misc/terms.xhtml). After 12 months, it is available under a Creative Commons License (AttributionNonCommercial 4.0 International), as described at http://creativecommons. org/licenses/by-nc/4.0/.
} 
Chattarji 2014) suggestive of the CS- being a neutral cue that lacks an emotional impact, or elevated by the CS- (albeit to a lesser extent than the CS+; Antunes and Moita 2010; Letzkus et al. 2011; Aizenberg and Geffen 2013; Botta et al. 2015; Abs et al. 2018), presumably due to fear generalization to the acoustic cues (Laxmi et al. 2003; Ghosh and Chattarji 2014). Thus, a paradigm in which animals learn both safety and fear in a cue-dependent manner with opposing behavioral expressions remains to be established. By using a discriminative AFC paradigm with multiple conditioning sessions and distinct acoustic features of CSs, here we demonstrate that C57BL/6J (B6) mice learn to discriminate between the CSand the $\mathrm{CS}+$, showing suppression of context-dependent freezing and disinhibited exploratory behaviors to the CS-, but cue-evoked freezing and rapid defensive behaviors to the CS+.

\section{Results}

\section{Acquisition of contextual and cued fear}

We first tested contextual and cued fear acquisition in a discriminative AFC paradigm with $\mathrm{B} 6$ mice. We performed conditioning for three consecutive days and retrieval testing for the next $2 \mathrm{~d}$, in one session per day (Fig. 1A). Each conditioning session during the first $3 \mathrm{~d}$ had four trials in which a white noise CS+ was paired with an electric footshock US, and four trials in which a $12 \mathrm{kHz}$ tone CS- was explicitly unpaired with the US; trials were executed in a pseudorandom order (Fig. 1A). The testing session on day 4 and day 5 had six trials in which the CS+ and the CS- were alternately presented in the absence of the US; with the session on day 4 performed in the same context of the conditioning chamber, and the session on day 5 performed in a novel spatial context (Fig. 1A). To avoid a masking effect on contextual fear acquisition, we omitted sessions for habituation to the chamber before conditioning. To assess fear strength in mice, we quantified body movements as the motion indices that are determined by pixels changed between successive frames in a recorded video, and measured time spent in freezing behavior defined as the motion indices of $0-2$ lasting more than or equal to $1 \mathrm{sec}$ (see Materials and Methods). In the first session of conditioning on day 1 , mice displayed exploratory behavior and little freezing during the time prior to the CS (PreCS) (Fig. 1B, Day 1, $2.4 \pm 0.9 \%, n=12$ ), indicating no intrinsic fear to the context of the chamber before learning. However, the freezing level during the PreCS was increased in most of the mice tested (10 of 12 mice) in the second session on day 2 (Fig. 1B). In the third conditioning session on day 3 , 9 mice exhibited an increase in the freezing levels from those on day 2 , and 4 mice showed extremely high levels ( $>60 \%)$ of freezing (Fig. 1B). On average, we found significantly higher level of freezing during the PreCS on day 2, and the high level was maintained on day 3 (Fig. $1 \mathrm{~B} ; P=$ 0.00739 (day 1 vs. day 2), $P=0.0105$ (day 1 vs. day 3 ), $P=0.826$ (day 2 vs. day 3 ), Bonferroni's test; $32.0 \pm 7.9 \%$ for day $2,38.9 \pm 10.2 \%$ for day $3, n=12$ ). These results indicate that the mice acquired contextual fear during conditioning.

To assess contextual fear memory, we examined the level of freezing at the beginning of the testing session on day 4 at which the mice were only subjected to the spatial context but not yet to the CSs on the day. The result showed a significantly higher level of freezing compared to the initial level on day 1 , but in the following testing session on day 5 when the spatial context of the chamber was changed, the freezing level dropped to the level before learning (Fig. 1C; $P=$ 0.00368 (day 1 vs. day 4), $P=0.00587$ (day 4 vs. day 5), $P=0.249$ (day 1 vs. day 5), Bonferroni's test; $1.4 \pm 1.0 \%$ for day 1 1st PreCS, $25.1 \pm 6.2 \%$ for day 41 st PreCS, $3.3 \pm 1.6 \%$ for day 51 st PreCS, $n=$ 12). The decrease of freezing level on day 5 is unlikely due to an attenuation of fear memories because the CS+ induced a marked increase of freezing level in the same session (Fig. $1 \mathrm{D} ; P=7.38 \times$ $10^{-6}, 7.8 \pm 2.5 \%$ for the PreCS,$+ 47.4 \pm$ $4.2 \%$ for the CS+, $n=12$ ), indicating the retention of cued fear memory to the CS+ on day 5. Taken together, the above results suggest that the mice acquire two features of conditioned fear, contextual fear and cued fear, during conditioning and retain the memory. 


\section{Learned safety for the CS-}

Interestingly, we also found that the freezing level was significantly lower during the tone CS- compared to that during the PreCS- in the testing session on day 4 (Fig. 2A; $P=0.00294,35.1 \pm 7.3 \%$ for the PreCS-, $10.1 \pm 4.2 \%$ for the CS,$- n=12$ ). In contrast, no difference was found for the noise $\mathrm{CS}+$ in this session (Fig. $2 \mathrm{~B} ; P=0.107$, $26.8 \pm 6.2 \%$ for the PreCS+, $39.9 \pm 5.0 \%$ for the CS+, $n=12$ ), although there was a marked difference on day 5 (Fig. 1D). To further assess cue-dependent effects, we analyzed changes in freezing levels from the PreCS period to the CS period in a trial-by-trial manner (see Materials and Methods) to subtract a component of contextual fear included in each trial. We found that 11 out of 12 mice exhibited negative changes from the PreCS- to the CS- in a trial on day 4 (Fig. 2C, CS- on day 4). In contrast, all of the mice tested displayed positive changes from the PreCS+ to the $\mathrm{CS}+$ in a trial on day 5 (Fig. $2 \mathrm{C}, \mathrm{CS}+$ on day 5 ). The averaged data showed a significantly negative change for the CS- as opposed to a positive change for the CS+ on both day 4 and day 5 (Fig. 2C; $P=0.000564,-22.1 \pm 5.1 \%$ for the CS $-, P=0.0457,14.9 \pm 8.1 \%$ for the CS+ (day 4$) ; P=0.0468$, $-6.8 \pm 3.7 \%$ for the CS $-, P=1.39 \times 10^{-6}, 38.4 \pm 4.4 \%$ for the CS+ (day 5 ), $n=12$ ). These results strongly suggest that contextual fear is suppressed by the CS- while cued fear is retrieved by the CS+.
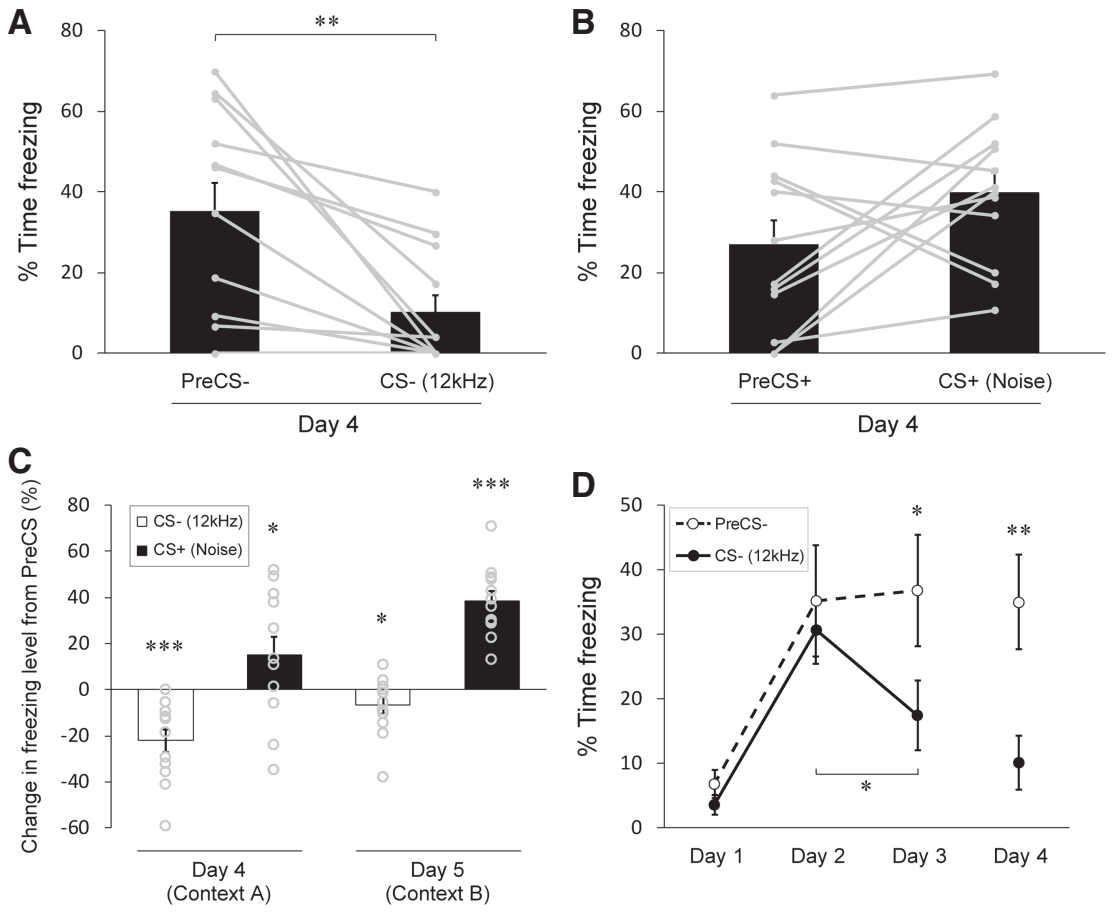

Figure 2. Learned suppression of contextual fear during the CS-. (A) Percent time spent freezing in trials with the CS- in the testing session with the conditioning context (day 4). The freezing level is markedly lower during the CS- than during the PreCS-, indicating inhibition of contextual fear during the CS-. (B) Percentage of time spent freezing in trials with the CS+ in the testing session on day 4 . On average, no significant change of the freezing level is detected during the CS+ comparerd to that during the PreCS+. (C) Changes in freezing levels represented as the percent time spent freezing during the CS subtracted by that during the corresponding PreCS of the trial. A negative change (i.e., reduction) of freezing levels for trials with the CS - and a positive change (i.e., enhancement) of freezing levels for trials with the CS + indicate opposing effects of the CSs on emotional responses. Note that the testing session on day 4 is performed in the conditioning context (context A), whereas the testing session on day 5 is carried out in a novel context (context B). (D) Comparison of freezing levels between the time during the PreCSand during the CS- across sessions. The freezing level during the CS- becomes significantly lower than that during the PreCS- on day 3 as well as on day 4. Gray dots with lines $(A, B)$ and circles $(C)$ represent the median of data sets from three trials for individual mice $(n=12$ mice). All data in bar graphs in $A-C$ and the line graph in $D$ are depicted as mean \pm SEM. $\left({ }^{*}\right) P<0.05,\left({ }^{* *}\right) P<0.01,\left({ }^{* * *}\right) P<0.001 . P$ values were obtained using a two-tailed paired $t$-test $(A, B, D)$ and a one-tailed paired $t$-test $(C)$.
In line with this observation, normal exploratory behaviors that are primarily represented as the motion indices ranging 3 to 1000 appeared more frequently during the CS- comtion of freezing), in the testing session on day 4 (Fig. $3 \mathrm{~A}, \mathrm{C} ; \mathrm{P}=$ $\pm 3.4 \%$ for the CS- (Motion index 3-1000); $P=0.905,1.6 \pm 1.0 \%$ for the PreCS-, $1.7 \pm 1.0 \%$ for the CS- (Motion index $>2000$ ), $n$ neutral cue that has no influence on behaviors but as a safety jumped the chat the mice sometimes rushed to the corner jumped in the chamber during the CS+ in the session on day 4. be reflected in the motion indices larger than 2000 during the (Fig. 3B, D; $P=0.00169,1.0 \pm 0.5 \%$ for the PreCS+, $5.0 \pm$ were observed in several short time epochs only during the CS+ ments with motion indices 3-1000 was decreased during the (1) $64.9 \pm 4.7 \%$ for the PreCS+, $47.6 \pm$ $2.9 \%$ for the CS+ (Motion index 3$1000), n=12$ ), while no significant difference was found in the motion indices of 0 between the PreCS+ and the CS+ in the session on day 4 (Fig. 3D; $P=0.122$, $29.0 \pm 5.7 \%$ for the PreCS+, $39.6 \pm 3.7 \%$ for the CS+ (Motion index 0), $n=12$ ). Thus, these behavioral differences during the CSs suggest emotional difference in the behavioral expressions elicited by the CS- and CS+.

To find whether the suppression of freezing observed during the tone CSwas acquired in conditioning sessions, we analyzed the difference of the freezing levels between the PreCS- and the CSacross three conditioning sessions (day 1-3). Interestingly, we observed that the freezing levels during the PreCS- and during the CS- showed similar increase on day 2 , and there was, therefore, no significant difference in the freezing level between the PreCS- and the CS-, as seen in day 1 (Fig. 2D; $P=0.189,6.8 \pm$ $2.2 \%$ for the PreCS-, $3.6 \pm 1.5 \%$ for the CS- (day 1); $P=0.580,35.2 \pm 8.7 \%$ for the PreCS-, $30.6 \pm 5.2 \%$ for the CS- (day $2), n=12$ ). However, the difference of the freezing levels between the PreCSand the CS- became significant on day 3 , due to a decrease of the freezing level during the CS- relative to that on day 2 (Fig. 2D; $P=0.0157,36.8 \pm 8.6 \%$ for the PreCS-, $17.5 \pm 5.4 \%$ for the CS- (day 3 ); $P=0.0415$ (day 2 vs. day 3 for the CS-), $n=12$ ). This result demonstrates that the mice acquired the CS- evoked suppression of contextual fear in the third conditioning session.

To test whether the suppression of freezing during the CS- is specific for pure tones, we performed the same conditioning experiment, with the $12 \mathrm{kHz}$ tone 

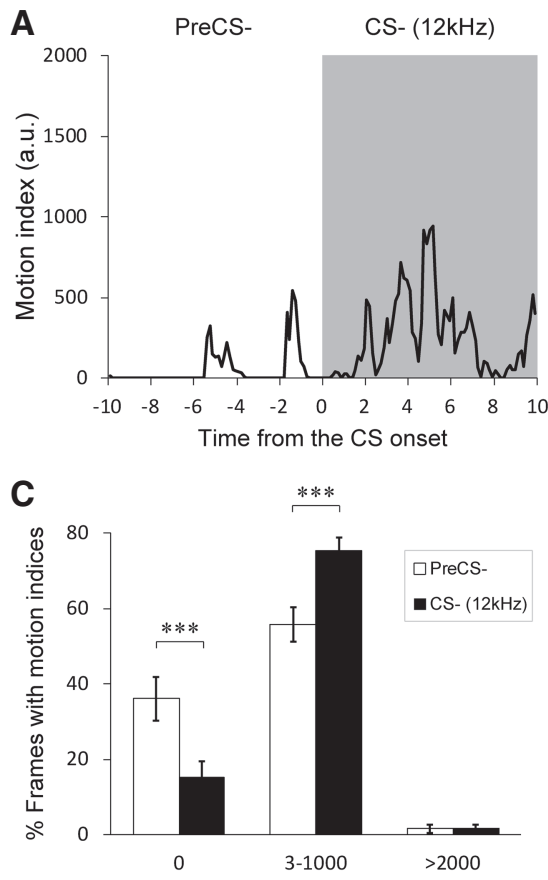

D
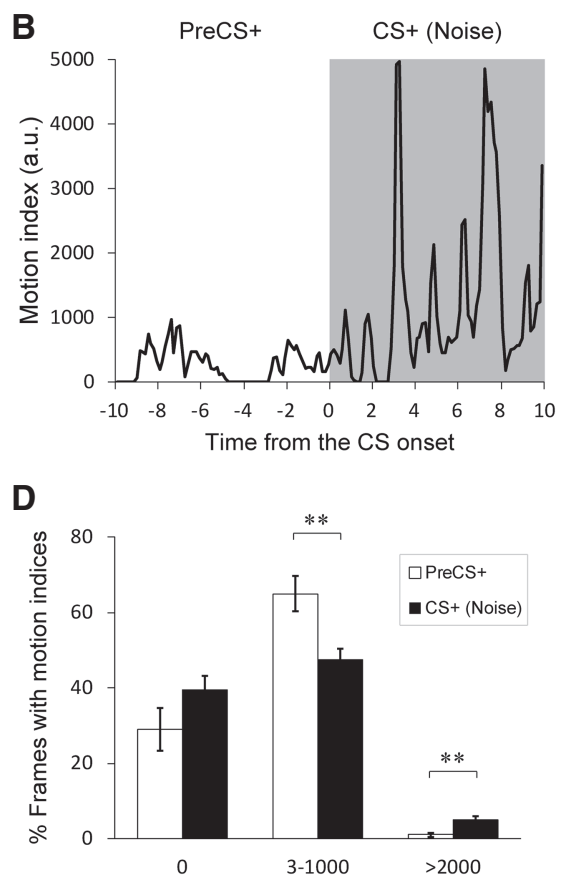

Figure 3. Distinct behavioral expressions in response to the $\mathrm{CS}$ - and the CS+. (A) A typical example of the motion indices during a trial with the $12 \mathrm{kHz}$ tone CS - (including the period of PreCS- for $10 \mathrm{sec}$ ) in a testing session on day 4 . The gray area represents the period of CS- presentation. The motion indices less than 1000 appear more frequently in the CS- period than in the PreCS- period. (B) A typical example of the motion indices during a trial with the white noise CS+ (including the period of PreCS+ for $10 \mathrm{sec}$ ) in a testing session on day 4 . The gray area represents the period of CS+ presentation. The motion indices more than 2000 appear in the CS- period. (C) Comparison of the appearance of mouse movements with different motion indices between the PreCS- and the tone CS- in the testing session on day 4 . The vertical axis represents the percent pixels (time) showing each motion index range $(0,3-1000$, or $>2000)$. The percentage of time with the motion index 0 is significantly lower during the CS- than during the PreCS-, while that with the motion indices ranging 3-1000 is significantly higher during the CS- than during the PreCS-. No significant difference is seen for the motion indices more than 2000. (D) Comparison of the appearance of mouse movements with different motion indices between the PreCS+ and the noise CS+ in the testing session on day 4. The percentage of time with the motion index more than 2000 is significantly higher during the CS+ than during the PreCS+, while that with the motion indices ranging 3-1000 is significantly lower during the CS- than during the PreCS-. No significant difference is seen for the motion index 0. All data in bar graphs are depicted as mean \pm SEM $\left(n=12\right.$ mice). $\left(^{* *}\right) P<0.01,\left({ }^{* * *}\right) P<0.001$. All $P$ values were obtained using a paired $t$-test.

and the white noise being oppositely assigned as the CS+ and CS-, respectively, for another group of mice. In accord with the previous results with the tone CS-, we found a significantly lower freezing level during the noise CS- compared to that during the PreCSon day 4 (Fig. $4 \mathrm{~A} ; P=0.0226,22.1 \pm 4.0 \%$ for the PreCS-, $11.1 \pm$ $3.8 \%$ for the $\mathrm{CS}-, \mathrm{n}=12$ ). We also found a significant reduction of contextual fear only for the noise CS-, but was not for the tone CS+ on day 4 (Fig. $4 \mathrm{~B}$, day 4 ; $P=0.0245,-13.1 \pm 3.9 \%$ for the CS- relative to the PreCS-, $P=0.0959,17.6 \pm 7.0 \%$ for the $\mathrm{CS}+$ relative to the PreCS,$+ n=12$ ), indicating that cue-evoked suppression of freezing is not limited to the $12 \mathrm{kHz}$ tone. In addition, the $12 \mathrm{kHz}$ tone $\mathrm{CS}+$, but not the noise $\mathrm{CS}-$, elevated freezing levels relative to the PreCS in a novel spatial context on day 5 (Fig. 4B, day 5; $P=0.134,-0.4 \pm 1.9 \%$ for the CS - relative to the PreCS-, $P=$ $0.000177,36.1 \pm 4.5 \%$ for the CS+ relative to the PreCS+, $n=12$ ), indicating that the $12 \mathrm{kHz}$ tone as the CS+ acts to retrieve fear memory in this protocol. Taken together, these results demonstrate that mice can learn the CS- and the CS+ as safety and threat signals, respectively, showing suppression of contextual fear for the CS- and expression of cued fear for the CS+ in our AFC paradigm with a noise and a tone as two acoustic CSs.

\section{No suppressive effect of the CS- on contextual fear in a paradigm with tone/ tone pair of the CSs}

To test whether the CS- shows a suppressive effect on contextual fear when two tones are used in our discriminative conditioning protocol, we carried out another experiment with 4 and $12 \mathrm{kHz}$ tones assigned as the CS+ and CS-, respectively, for a different group of mice. Although 4 of 12 mice displayed a large decrease (more than 10\% differences compared to that during the PreCS-) in freezing levels during the CS- in the testing session on day 4 , the remaining mice (8 of 12 mice) did not show such reductions consistently (Fig. 5A). On average, no significantly lower level of freezing was observed during the CS- compared to that during the PreCS- on day 4 (Fig. 5A; $P=0.506,15.7 \pm 4.1 \%$ for the PreCS-, $20.5 \pm 5.4 \%$ for the CS-, $n=12$ ). This result indicates no apparent effect of the CS- on contextual fear in this protocol. However, we found markedly higher level of freezing during the $\mathrm{CS}+$ relative to that during the PreCS+ (Fig. 5B; $P=0.000158$, $10.8 \pm 4.0 \%$ for the PreCS+, $47.5 \pm 5.6 \%$ for the CS+, $n=12$ ), and a significant difference between the CS- and the CS+ in freezing level changes from the PreCS period to the $\mathrm{CS}$ period on day 4 (Fig. $5 \mathrm{C} ; P=$ $0.000443,1.8 \pm 6.0 \%$ for the CS- relative to the PreCS-, $35.2 \pm 5.9 \%$ for the CS+ relative to the PreCS+, $n=12$ ), indicating that the mice discriminated the two tone CSs. We also observed a positive change in the freezing level relative to that during the PreCS only during the $4 \mathrm{kHz}$ tone CS+ but not during the $12 \mathrm{kHz}$ tone CS- in a novel spatial context on day 5 (Fig. 5D; $P=0.319,1.3 \pm 2.6 \%$ for the CS- relative to the PreCS-, $P=1.39 \times 10^{-7}, 49.0 \pm$ $4.4 \%$ for the CS+ relative to the PreCS+, $n=12$ ), indicating cued fear memory for the CS+. These results suggest that mice fail to express safety behavior during the CS- in our AFC paradigm with a tone/tone pair of CSs, although they can discriminate between the two CSs.

\section{Discussion}

In this study, we designed a discriminative AFC paradigm with 3-d conditioning sessions in which a pair of noise and tone pulses was used as CSs, and demonstrated that B6 mice learned contextual fear and suppression of contextual fear in response to the CS-, as well as cue-specific fear to the CS+. Our study provides an experimental model of discriminative learning and memory for safety versus fear that is useful for elucidating the neural mechanisms of emotion regulation and dysregulation.

Two types of safety conditioning paradigms have been classically utilized in rodent studies: a single-cue paradigm and a dualcues paradigm (Hammond 1967; Rescorla 1969; Rogan et al. 2005; Kong et al. 2014). The CS in the single-cue paradigm exerts an inhibitory effect on contextual fear (Rogan et al. 2005; Pollak 
A

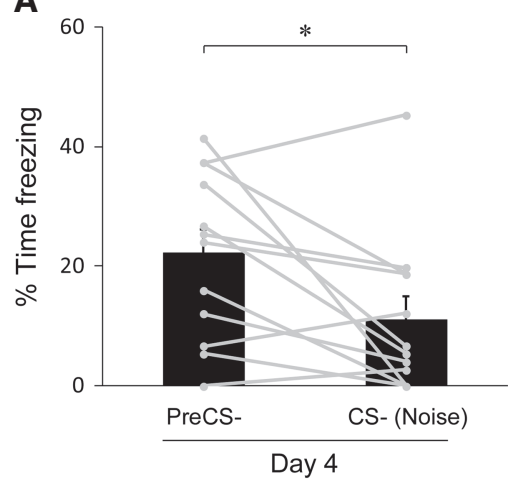

B

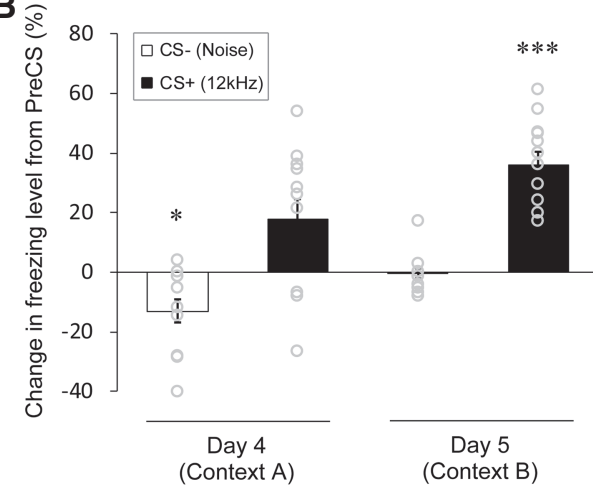

Figure 4. Discriminative safety and fear learning in a paradigm with the tone CS+ and the noise CS-. (A) Percent time spent freezing in trials with the noise CS- in the testing session with the conditioning context (day 4). The freezing level is significantly lower during the CS- than during the PreCS-, indicating inhibition of contextual fear is caused by the noise CS-. $(B)$ Changes in freezing levels represented as the percent time spent freezing during the CS subtracted by that during the corresponding PreCS of the trial. Opposite changes in freezing levels between the CS- on day 4 (decrease) and the CS+ on day 5 (increase) indicate safety specific for the noise CS- and fear specific for the tone CS+. Gray dots with lines $(A)$ and circles $(B)$ represent the median of data sets from three trials for individual mice $(n=12$ mice). All data in bar graphs are depicted as mean \pm SEM. $\left.{ }^{*}\right) P<0.05,\left({ }^{* * *}\right) P<0.001$. $P$ values were obtained using a two-tailed paired $t$-test $(A)$ and a one-tailed paired $t$-test $(B)$.

et al. 2008, 2010), while the CS- in the dual-cues paradigm have an attenuating effect on cued fear induced by the CS+ (Hammond 1967; Rescorla 1969; Christianson et al. 2012; Sangha et al., 2013; Kong et al. 2014; Foilb et al. 2016). In the present study, we integrated the evaluation of safety learning based on a suppression of contextual fear, classically used in single-cue paradigms (Rogan et al. 2005; Pollak et al. 2008, 2010), into the auditory discriminative paradigm that involves two acoustic cues. This allowed us to assess not only safety for the CS- but also fear for the CS+ in the same manner as the CS's effect on contextual fear.

Many rodent studies with discriminative AFC paradigms used two pure tones with different frequencies (Antunes and Moita 2010; Aizenberg and Geffen 2013; Ghosh and Chattarji 2014), two frequency-modulated sweeps with the same frequency range but opposite sweep directions (Letzkus et al. 2011; Abs et al. 2018), or a pair of a tone and a noise (Courtin et al. 2014; Botta et al. 2015) as the CSs. These examinations, however, resulted in either an increase in the freezing level even during the CS- compared to that during the PreCS or the baseline level (Antunes and Moita 2010; Letzkus et al. 2011; Aizenberg and Geffen 2013; Botta et al. 2015; Abs et al. 2018), or unaltered freezing levels during the CS- (Ghosh and Chattarji 2014) (except for Courtin et al. 2014 which made no statistical comparison between pre- and postconditioning for the CS-), although there was a significant difference in freezing level between the CS- and CS+ after learning (Antunes and Moita 2010; Letzkus et al. 2011; Aizenberg and Geffen 2013; Courtin et al. 2014; Ghosh and Chattarji 2014; Botta et al. 2015; Abs et al. 2018).

There are several potential factors that affect specification and generalization of fear in discriminative AFC paradigms. First, when the physical properties of the two sound stimuli are similar, fear generalization to the sound stimuli occurs more likely (Aizenberg and Geffen 2013). In our present study, we chose a white noise and a tone delivered at different pulse rates as sound CSs to allow mice to discriminate more easily the two stimuli. As a result, the mice did not show generalized fear to the CS- but displayed a safety response selectively to the CS- after learning. We also found that a pure tone and a white noise as the CS- had a similar effect on conditioned safety in our discriminative conditioning paradigm. On the other hand, we found no learned suppression of contextual fear in response to the CS- in the same paradigm but with a tone/tone pair of the CSs, which is consistent with the previous studies described above. Second, to detect a suppressive effect of the CS- on contextual fear, it is important for mice to be subjected to the CS- in the presence of relatively high contextual fear that is caused by fear generalization. However, preconditioning habituation protocols can attenuate generalized fear to the spatial context that can be otherwise enhanced by the US during conditioning (see below). In our current protocol, we omitted the habituation session to avoid its masking effect on fear generalization. As we expected, mice showed a substantial level of contextual fear in the second and third sessions of conditioning and also in the testing session with the same spatial context (Figs. 1B, 2D). Importantly, all the above previous studies performed retrieval testing only in a cage that differs from the one used for conditioning (i.e., a novel context) (Antunes and Moita 2010; Letzkus et al. 2011; Aizenberg and Geffen 2013; Courtin et al. 2014; Ghosh and Chattarji 2014; Botta et al. 2015; Abs et al. 2018). The context change for testing in those studies may have masked the suppressive effect of the $\mathrm{CS}$ - on contextual fear. Actually, we found a decreased freezing level during the PreCS in the testing session with a novel context (day 5 ) in our conditioning paradigm. We cannot rule out the possibility that this reduction was caused in part by an attenuation of contextual fear memory or extinction learning during the testing session on day 4 . Nevertheless, we found cued fear selective to the CS+, but not to the CS-, in a novel spatial context (Figs. 1D, 2C day 5), indicating the retention of fear memory on day 5. Additionally, the total number of trials with US as well as the intensity of the US can affect fear generalization to the context (Laxmi et al. 2003; Baldi et al. 2004) and also to the CS- (Laxmi et al. 2003; Antunes and Moita 2010; Ghosh and Chattarji 2014), and can thus determine the level of fear specificity to the CS+. Indeed, we found a high level of freezing during the CS- which was comparable to that during the PreCS- in the second conditioning session, but suppression of freezing during the CS- appeared in the third session (Fig. 2D, day 2 vs. day 3), although the effect of the CS+ on freezing is likely masked by contextual fear in these sessions. An optimal set of conditioning parameters might therefore be required for the successful detection of contextual fear, its suppression by the CS-, and cued fear to the CS+ in discriminative fear conditioning paradigms.

Besides the suppression of freezing, we also observed increased occurrence of normal exploratory behaviors during the CS- in conditioned mice, in stark contrast to defensive behaviors caused by the CS+ (Fig. 3). This result suggests the potential overlap between safety and reward. In support of this notion, safety learning enhanced CS- evoked responses in the striatum (Rogan et al. 2005) and the basal amygdala (Sangha et al. 2013), the brain regions known to regulate behaviors motivated by reward (Balleine et al. 2007; Ambroggi et al. 2008; Haber and Knutson 2010; Namburi et al. 2015). A recent study further demonstrated the involvement of dopamine signaling in the basolateral amygdala in learned safety ( $\mathrm{Ng}$ et al. 2018). Further studies will be needed to address whether the CS- acts as a rewarding stimulus in our conditioning paradigm. Nevertheless, our discriminative AFC paradigm serves as an experimental model for discriminatory 
A
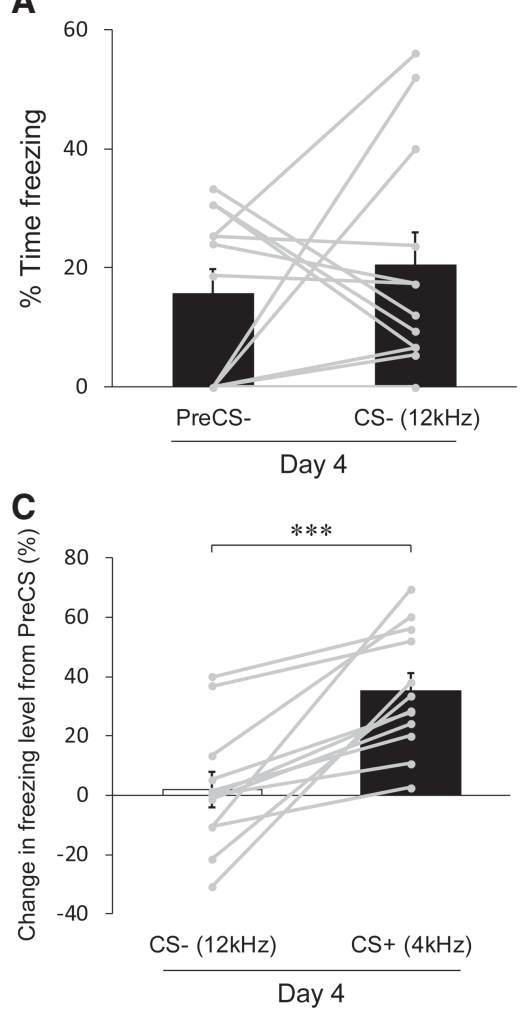

B

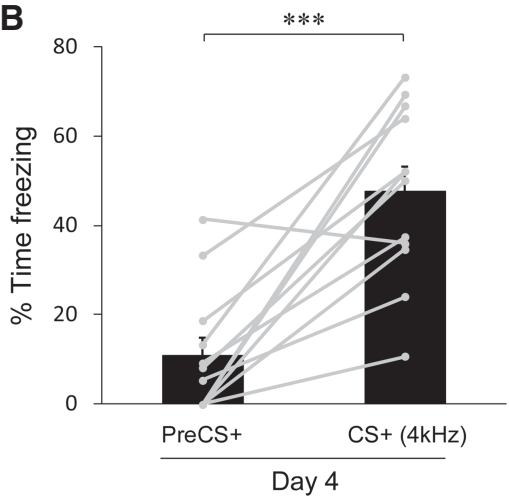

D

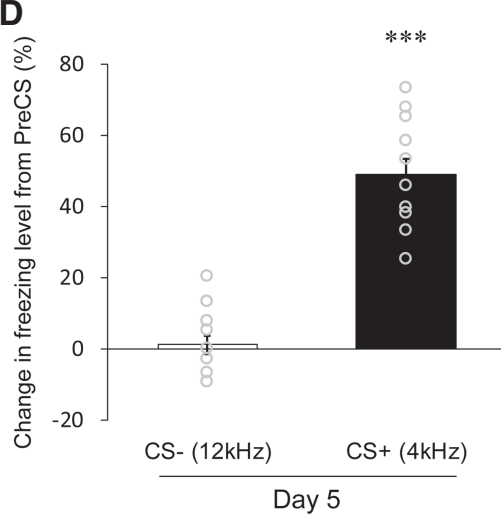

Figure 5. Discriminative fear but not safety learning in a paradigm with tone/tone pair of the CSs. (A) Percentage of time spent freezing in trials with the $12 \mathrm{kHz} \mathrm{CS}-$ in the testing session with the conditioning context (day 4). No significant difference in the freezing level on average is found between the CS- and the PreCS-, indicating no apparent effect of the CS- on contextual fear in this paradigm. $(B)$ Percentage of time spent freezing in trials with the $4 \mathrm{kHz} C \mathrm{CS}+$ on day 4 . A significantly higher level of freezing is found for the CS+ compared to the PreCS+. (C) Comparison of the changes in freezing levels from the PreCS period to the CS period on day 4. A significant difference of the changes in freezing levels during the CSs indicates discriminatory responses to the tone CSs. (D) A positive change in the freezing level is found for the $4 \mathrm{kHz} C S+$ but not for the $12 \mathrm{kHz} C S$ - in the testing session with a novel context (day 5), indicating cued fear selective to the $4 \mathrm{kHz} C S+$. Gray dots with lines $(A-C)$ and circles $(D)$ represent the median of data sets from three trials for individual mice $(n=12$ mice). All data in bar graphs are depicted as mean \pm SEM. $\left(^{* * *}\right) P<0.001$. P values were obtained using a two-tailed paired $t$-test $(A-C)$ and a one-tailed paired $t$-test $(D)$.

behaviors in mice that depend on emotional valence of the acoustic cues.

\section{Materials and Methods}

\section{Animals}

Male B6 (6-10 wk old) mice used for the experiments were purchased from Japan SLC (Shizuoka, Japan) or bred in our facility. All animals were individually housed and maintained for at least $2 \mathrm{~d}$ until experiments in a breeding room on a $12 \mathrm{~h}$ light-dark cycle with food and water available ad libitum. All experiments were approved by the Committee for Animal Experiments of Kumamoto University, and performed in accordance with the Guidelines for Use of Animals in Experiments of Kumamoto University.

\section{Fear conditioning apparatus}

All type of fear conditioning and testing experiments were performed in fear conditioning chambers (MED-AFC-M1, MedAssociates) individually placed in sound attenuating cubicles (NSB-B101) with an exhaust fan on the wall and a room lamp on the ceiling. The conditioning chamber was equipped with a chamber speaker and a metal grid floor connected with a programable shocker (ENV-414S, Med-Associates). A video camera was placed outside of a clear acrylic wall of the chamber. Scrambled footshock unconditioned stimuli (US; $0.6 \mathrm{~mA}, 2 \mathrm{sec}$ ) were generated by the shock generator and delivered through the grids of the floor. FreezeFrame 3 (Actimetrics) software was used for sound stimulus configuration, temporal control of electric shocks, video tracking, and quantification of locomotion and freezing behavior. "Significant Motion Pixel" analysis in the software was chosen to quantify animal movements (Kopec et al. 2007). The frame rate of the video recording was 7.5 frames per second. Before experiments the grid floor with an excreta tray was washed and dried, and the inside walls of conditioning chambers were cleaned with $50 \mathrm{ppm}$ sodium hypochlorite. Additionally, the doors of sound attenuating cubicles and conditioning chambers were opened and exhaust fans were turned on for ventilation until experiments. For testing with a novel context, a gray acrylic triangle roof and a white acrylic floor were used (see below).

\section{Conditioning and testing protocols}

All experiments were conducted within the light cycle. A discriminative fear conditioning paradigm consisted of 3-d conditioning (day 1 to 3 ) and 2-d testing (day 4 and 5) sessions with a conditioning context (context A) on day 1 to 4 and a novel context (context B) on day 5 (Fig. $1 \mathrm{~A})$. In a conditioning session, four trials with conditioned stimuli (CS+) paired with the US and four trials with conditioned stimuli (CS-) explicitly unpaired with the US were performed in a pseudorandom order, with the inter-trial interval randomly selected from four intervals: 60 , 70,80 , and $90 \mathrm{sec}$. Each conditioning session lasted for $600 \mathrm{sec}$, and the next session started $24 \pm 2 \mathrm{~h}$ later (one session in a day). CS+ was a $10 \mathrm{sec}$ white noise (125 msec pulse delivered at $4 \mathrm{~Hz}$ ), and CS- was a $10 \mathrm{sec} 12 \mathrm{kHz}$ tone $(250 \mathrm{msec}$ pulse delivered at $2 \mathrm{~Hz})$. In the second experiments, the tone and noise pulse trains were assigned as the CS+ and CS-, respectively. A $4 \mathrm{kHz}$ and a $12 \mathrm{kHz}$ tone pulse trains (both of which were delivered as $250 \mathrm{msec}$ pulses at 2 $\mathrm{Hz}$ ) were assigned as the $\mathrm{CS}+$ and $\mathrm{CS}-$, respectively, in the third experiments. The CS was presented at $80 \mathrm{~dB}$ sound pressure level. The US was delivered during the last 2 sec of the CS+, so that they coterminated. Mice were not habituated to the conditioning chamber and sound stimuli before conditioning to avoid a masking effect on contextual fear acquisition and a potential effect on reversal learning for the CS+, respectively. For testing sessions on day 4 and 5, three pairs of trials with the CS+ and CS- were performed in an alternate order with the same inter-trial interval range as conditioning sessions in the absence of the US. Each testing session lasted for $450 \mathrm{sec}$. For testing with a novel context (context B) on day 5 , the session was carried out in the conditioning chamber whose grid floor was covered with a white acrylic plate and a gray acrylic triangle roof. Soon after each session, mice were returned to their home cage and transferred to the breeding room.

\section{Data analysis}

Freezing was defined as the motion index less than or equal to 2 with the bout duration more than or equal to $1 \mathrm{sec}$. To evaluate behavioral responses to the CS, the percentage of time spent in 
freezing during CS (CS+/CS-) and during the 10 sec prior to the CS (PreCS+/PreCS-) was calculated for each trial and each session. The final 2 sec of both CS+ and CS- in the 3-d conditioning sessions was excluded from analysis to eliminate the effect of the US. To compare cue-evoked responses among animals, freezing level during the PreCS was subtracted from that during the CS for each trial to eliminate the influence of contextual fear. To eliminate a potential bias for the freezing levels caused by incidental extreme outliers that can appear in a trial of the session, median values were sampled from the data sets of four trials (Day 1-3) or three trials (Day 4 and 5) in each session for the PreCS+, CS+, PreCS-, CS-, or trial-by-trial subtraction of the PreCS from the corresponding CS for each animal tested, and averaged by the number of animals for each session. For the comparison of the PreCS among days (day 1-3, Fig. 1B), medians from eight trials (including four trials with the CS+ and four trials with the CS-) were extracted for each animal, and averaged by the number of animals for each session. For the assessment of contextual fear memory, data were sampled from the PreCS period of the first trial in each session (Fig. 1C). For statistical analysis, the percentages of time spent in freezing were compared using a paired $t$-test except for multiple comparisons among 3-d sessions with the Bonferroni's multiple comparison test. Significance of negative or positive changes in freezing level from the PreCS to the CS was analyzed using a onetailed $t$-test. Data are expressed as means \pm standard error of the mean (SEM). Differences were considered statistically significant if $P<0.05$.

\section{Competing interest statement}

The authors declare that this study was conducted in the absence of any financial or commercial relationships that could be construed as a potential conflict of interest.

\section{Acknowledgments}

We thank the Kumamoto University Institute of Resource Development and Analysis Center for Animal Resources and Development for animal care and management of fear conditioning devices. This work was supported by Grant-in-Aid for Scientific Research (C) (16K10218) and partly by Grant-in-Aid for Scientific Research on Innovative Areas (17H05749) from the Japan Society for the Promotion of Science.

\section{References}

Abs E, Poorthuis RB, Apelblat D, Muhammad K, Pardi MB, Enke L, Kushinsky D, Pu DL, Eizinger MF, Conzelmann KK, et al. 2018. Learning-related plasticity in dendrite-targeting layer 1 interneurons. Neuron 100: 684-699. doi:10.1016/j.neuron.2018.09.001

Aizenberg M, Geffen MN. 2013. Bidirectional effects of aversive learning on perceptual acuity are mediated by the sensory cortex. Nat Neurosci 16: 994-996. doi:10.1038/nn.3443

Ambroggi F, Ishikawa A, Fields HL, Nicola SM. 2008. Basolateral amygdala neurons facilitate reward-seeking behavior by exciting nucleus accumbens neurons. Neuron 59: 648-661. doi:10.1016/j.neuron.2008 .07 .004

Antunes R, Moita MA. 2010. Discriminative auditory fear learning requires both tuned and nontuned auditory pathways to the amygdala. J Neurosci 30: 9782-9787. doi:10.1523/JNEUROSCI.1037-10.2010

Baldi E, Lorenzini CA, Bucherelli C. 2004. Footshock intensity and generalization in contextual and auditory-cued fear conditioning in the rat. Neurobiol Learn Mem 81: 162-166. doi:10.1016/j.nlm.2004.02.004

Balleine BW, Delgado MR, Hikosaka O. 2007. The role of the dorsal striatum in reward and decision-making. J Neurosci 27: 8161-8165. doi:10.1523/ JNEUROSCI.1554-07.2007

Botta P, Demmou L, Kasugai Y, Markovic M, Xu C, Fadok JP, Lu T, Poe MM, $\mathrm{Xu}$ L, Cook JM, et al. 2015. Regulating anxiety with extrasynaptic inhibition. Nat Neurosci 18: 1493-1500. doi:10.1038/nn.4102

Braga RJ, Reynolds GP, Siris SG. 2013. Anxiety comorbidity in schizophrenia. Psychiatry Res 210: $1-7$. doi:10.1016/j.psychres.2013.07 .030

Christianson JP, Fernando ABP, Kazama AM, Jovanovic T, Ostroff LE, Sangha S. 2012. Inhibition of fear by learned safety signals: a mini-symposium review. J Neurosci 32: 14118-14124. doi:10.1523/ JNEUROSCI.3340-12.2012

Courtin J, Chaudun F, Rozeske RR, Karalis N, Gonzalez-Campo C, Wurtz H, Abdi A, Baufreton J, Bienvenu TCM, Herry C. 2014. Prefrontal parvalbumin interneurons shape neuronal activity to drive fear expression. Nature 505: 92-96. doi:10.1038/nature 12755

Dunsmoor JE, Paz R. 2015. Fear generalization and anxiety: behavioral and neural mechanisms. Biol Psychiatry 78: 336-343. doi:10.1016/j.biopsych .2015 .04 .010

Etkin A, Wager TD. 2007. Functional neuroimaging of anxiety: a metaanalysis of emotional processing in PTSD, social anxiety disorder, and specific phobia. Am J Psychiatry 164: 1476-1488. doi:10.1176/appi.ajp 2007.07030504

Foilb AR, Flyer-Adams JG, Maier SF, Christianson JP. 2016. Posterior insular cortex is necessary for conditioned inhibition of fear. Neurobiol Learn Mem 134: 317-327. doi:10.1016/j.nlm.2016.08.004

Gerlai R. 1998. Contextual learning and cue association in fear conditioning in mice: a strain comparison and a lesion study. Behav Brain Res 95: 191203. doi:10.1016/S0166-4328(97)00144-7

Ghosh S, Chattarji S. 2014. Neuronal encoding of the switch from specific to generalized fear. Nat Neurosci 18: 112-120. doi:10.1038/nn.3888

Haber SN, Knutson B. 2010. The reward circuit: linking primate anatomy and human imaging. Neuropsychopharmacology 35: 4-26. doi:10.1038/ npp.2009.129

Hammond LJ. 1967. A traditional demonstration of the properties of Pavlovian inhibition using differential CER. Psychon Sci 9: 65-66. doi:10 .3758/BF03330761

Holt DJ, Coombs G, Zeidan MA, Goff DC, Milad MR. 2012. Failure of neural responses to safety cues in schizophrenia. Arch Gen Psychiatry 69: 893903. doi:10.1001/archgenpsychiatry.2011.2310

Jovanovic T, Kazama A, Bachevalier J, Davis M. 2012. Impaired safety signal learning may be a biomarker of PTSD. Neuropharmacology 62: 695-704 doi:10.1016/j.neuropharm.2011.02.023

Kheirbek MA, Klemenhagen KC, Sahay A, Hen R. 2012. Neurogenesis and generalization: a new approach to stratify and treat anxiety disorders. Nat Neurosci 15: 1613-1620. doi:10.1038/nn.3262

Kiran C, Chaudhury S. 2016. Prevalence of comorbid anxiety disorders in schizophrenia. Ind Psychiatry J 25: 35. doi:10.4103/0972-6748.196045

Kong E, Monje FJ, Hirsch J, Pollak DD. 2014. Learning not to fear: neural correlates of learned safety. Neuropsychopharmacology 39: 515-527. doi:10.1038/npp.2013.191

Kopec CD, Kessels HWHG, Bush DEA, Cain CK, LeDoux JE, Malinow R. 2007. A robust automated method to analyze rodent motion during fear conditioning. Neuropharmacology 52: 228-233. doi:10.1016/j .neuropharm.2006.07.028

Laxmi TR, Stork O, Pape HC. 2003. Generalisation of conditioned fear and its behavioural expression in mice. Behav Brain Res 145: 89-98. doi:10 .1016/S0166-4328(03)00101-3

Letzkus JJ, Wolff SBE, Meyer EMM, Tovote P, Courtin J, Herry C, Lüthi A. 2011. A disinhibitory microcircuit for associative fear learning in the auditory cortex. Nature 480: 331-335. doi:10.1038/nature10674

Namburi P, Beyeler A, Yorozu S, Calhoon GG, Halbert SA, Wichmann R, Holden SS, Mertens KL, Anahtar M, Felix-Ortiz AC, et al. 2015. A circuit mechanism for differentiating positive and negative associations. Nature 520: $675-678$. doi: $10.1038 /$ nature 14366

$\mathrm{Ng}$ KH, Pollock MW, Urbanczyk PJ, Sangha S. 2018. Altering D1 receptor activity in the basolateral amygdala impairs fear suppression during a safety cue. Neurobiol Learn Mem 147: 26-34. doi:10.1016/j.nlm.2017.11.011

Pollak DD, Monje FJ, Zuckerman L, Denny CA, Drew MR, Kandel ER. 2008. An animal model of a behavioral intervention for depression. Neuron 60: 149-161. doi:10.1016/j.neuron.2008.07.041

Pollak DD, Monje FJ, Lubec G. 2010. The learned safety paradigm as a mouse model for neuropsychiatric research. Nat Protoc 5: 954-962. doi:10 $.1038 /$ nprot.2010.64

Rescorla RA. 1969. Pavlovian conditioned inhibition. Psychol Bull 72: 77-94. doi:10.1037/h0027760

Rogan MT, Leon KS, Perez DL, Kandel ER. 2005. Distinct neural signatures for safety and danger in the amygdala and striatum of the mouse. Neuron 46: 309-320. doi:10.1016/j.neuron.2005.02.017

Sangha S, Chadick JZ, Janak PH. 2013. Safety encoding in the basal amygdala. J Neurosci 33: 3744-3751. doi:10.1523/JNEUROSCI.3302-12.2013

van Steensel FJA, Bögels SM, Perrin S. 2011. Anxiety disorders in children and adolescents with autistic spectrum disorders: a meta-analysis. Clin Child Fam Psychol Rev 14: 302-317. doi:10.1007/s10567-011-0097-0

White SW, Mazefsky CA, Dichter GS, Chiu PH, Richey JA, Ollendick TH. 2014. Social-cognitive, physiological, and neural mechanisms underlying emotion regulation impairments: understanding anxiety in autism spectrum disorder. Int J Dev Neurosci 39: 22-36. doi:10.1016/j .ijdevneu.2014.05.012

Received March 28, 2019; accepted in revised form May 25, 2019. 


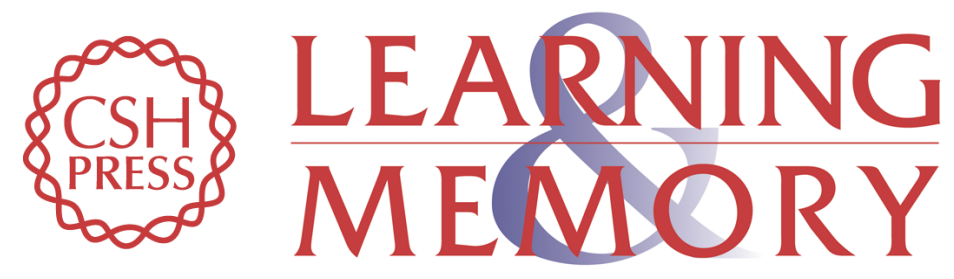

\section{Cue-dependent safety and fear learning in a discriminative auditory fear conditioning paradigm in the mouse}

Makoto Takemoto and Wen-Jie Song

Learn. Mem. 2019, 26:

Access the most recent version at doi:10.1101/Im.049577.119

References This article cites 34 articles, 4 of which can be accessed free at:

http://learnmem.cshlp.org/content/26/8/284.full.html\#ref-list-1

Creative This article is distributed exclusively by Cold Spring Harbor Laboratory Press for the

Commons

first 12 months after the full-issue publication date (see

License http://learnmem.cshlp.org/site/misc/terms.xhtml). After 12 months, it is available under a Creative Commons License (Attribution-NonCommercial 4.0 International), as described at http://creativecommons.org/licenses/by-nc/4.0/.

Email Alerting Receive free email alerts when new articles cite this article - sign up in the box at the Service top right corner of the article or click here. 